\title{
Impacto da osteoartrose de joelho na capacidade funcional e qualidade de vida de pacientes atendidos em um município de Pernambuco, Brasil
}

\author{
Impact of knee osteoarthrosis on the functional capacity and quality of life of patients treated in a municipality \\ of Pernambuco, Brazil \\ Impacto de la osteoartritis de rodilla en la capacidad funcional y calidad de vida de pacientes atendidos en un \\ Municipio de Pernambuco, Brasil
}

Ruan Emerson RODRIGUES ${ }^{1}$

Paulo Henrique Meira DUARTE ${ }^{2}$

Cleyton Ânderson Leite FEITOSA ${ }^{3}$

${ }^{1}$ Centro Universitário de João Pessoa, UNIPÊ 58053-000 João Pessoa-PB, Brasil

${ }^{2}$ Faculdade de Integração do Sertão, FIS 56909-205 Serra Talhada-PE, Brasil

\begin{abstract}
Resumo
Introdução: A osteoartrose é definida como uma patologia de aspecto crônico-degenerativa que agride a cartilagem articular, gerando dor, edema, rigidez matinal, redução da amplitude de movimento e influenciando diretamente na capacidade funcional e em aspectos da qualidade de vida. Objetivo: Investigar o perfil sociodemográfico e o impacto da osteoartrose de joelho na capacidade funcional e qualidade de vida dos pacientes residentes da Cidade de Serra Talhada - PE. Material e método: Trata-se de um estudo descritivo, de corte transversal e natureza quantitativa, que foi realizado na rede municipal de fisioterapia na cidade de Serra Talhada em Pernambuco. Foram empregados os seguintes instrumentos: questionário sociodemográfico, a escala visual analógica para mensurar a dor, o Western Ontario McMaster Universities Osteoarthritis Index para avaliar a capacidade funcional e o Short-Form 36 para analisar a qualidade de vida. Resultado: A amostra foi composta por 26 indivíduos com média de idade de 63,4 anos, constituída de 80,7\% de mulheres e 19,3\% de homens. As atividades com maior limitação física foram calçar meias e subir escadas e as menos afetadas foram caminhar e fazer compras. Os aspectos de qualidade de vida mais acometidos foram a capacidade funcional e o estado geral de saúde. Conclusão: Foi verificado que indivíduos com osteoartrose de joelho apresentam dor intensa nessa articulação, com limitação funcional para determinadas atividades cotidianas afetando diretamente alguns aspectos da qualidade de vida.
\end{abstract}

Descritores: Osteoartrite; Joelho; Qualidade de Vida; Classificação Internacional de Funcionalidade, Incapacidade e Saúde.

\section{Abstract}

Osteoarthrosis is defined as a chronic-degenerative disease that affects the articular cartilage, causing pain, edema, morning stiffness, reduced range of motion and directly influencing functional capacity and aspects of quality of life. Objective: To investigate the sociodemographic profile and the impact of knee osteoarthrosis on the functional capacity and quality of life of patients living in the city of Serra Talhada - PE. Material and method: This is a descriptive, cross-sectional and quantitative study that was carried out in the municipal physiotherapy network in the city of Serra Talhada in Pernambuco. The following instruments were used: socio-demographic questionnaire, visual analogue scale to measure pain, Western Ontario McMaster University Osteoarthritis Index to evaluate functional capacity and ShortForm 36 to analyze quality of life. Results: The sample consisted of 26 individuals with a mean age of 63.4 years, comprising $80.7 \%$ of women and $19.3 \%$ of men. The activities with the greatest physical limitation were shoeing socks and climbing stairs and the least affected were walking and shopping. The most affected aspects of quality of life were functional capacity and general health status. Conclusion: It was verified that individuals with knee osteoarthrosis present intense pain in this joint, with functional limitation for certain daily activities directly affecting some aspects of quality of life.

Descriptors: Osteoarthritis; Knee; Quality of Life; International Classification of Functioning, Disability and Health.

\section{Resumen}

Introducción: La osteoartrosis se define como una patología de aspecto crónico-degenerativa que afecta el cartílago articular, generando dolor, edema, rigidez matinal, reducción de la amplitud de movimiento e influenciando directamente en la capacidad funcional y en aspectos de la calidad de vida. Objetivo: Investigar el perfil sociodemográfico y el impacto de la osteoartritis de rodilla en la capacidad funcional y calidad de vida de los pacientes residentes de la Ciudad de Serra Talhada - PE. Material y método: Se trata de un estudio descriptivo, de corte transversal y naturaleza cuantitativa, que fue realizado en la red municipal de fisioterapia en la ciudad de Serra Talada en Pernambuco. Se utilizaron los siguientes instrumentos: cuestionario sociodemográfico, la escala visual analógica para medir el dolor, el Western Ontario McMaster Universities Osteoarthritis Index para evaluar la capacidad funcional y el Short-Form 36 para analizar la calidad de vida. Resultado: La muestra fue compuesta por 26 individuos con media de edad de 63,4 años, constituida de $80,7 \%$ de mujeres y $19,3 \%$ de hombres. Las actividades con mayor limitación física fueron calzar medias y subir escaleras y las menos afectadas fueron caminar y hacer compras. Los aspectos de calidad de vida más acometidos fueron la capacidad funcional y el estado general de salud. Conclusión: Se verificó que los individuos con artrosis de rodilla presentan dolor intenso en esa articulación, con limitación funcional para determinadas actividades cotidianas afectando directamente algunos aspectos de la calidad de vida.

Descriptores: Osteoartritis; Rodilla; Calidad de Vida; Clasificación Internacional del Funcionamiento, de la Discapacidad y de la Salud.

\section{INTRODUÇÃO}

A osteoartrose (OA) é um processo
patológico importante de aspecto crônicodegenerativo, considerado como um problema grave de saúde pública, sendo também uma doença de frequência importante na população mundial ${ }^{1}$. Acomete de forma comum as articulações da mão, coluna vertebral, quadril e especialmente o joelho, apresentando-se em $37 \%$ dos casos ${ }^{2}$.

A OA tem grande incidência, atingindo aproximadamente $20 \%$ da população mundial com idade acima de 60 anos $^{3}$, e ainda, poderá ser a sétima morbidade de maior incidência no mundo até $2020^{4}$.
A literatura mostra que a $\mathrm{AO}$ acomete ambos os sexos, entretanto a maior prevalência encontra-se em mulheres e na faixa etária acima de 60 anos $^{5}$.

A AO do joelho não acomete apenas a cartilagem hialina, mais também prejudica as estruturas periarticulares como o osso subcondral ${ }^{6}$, causando de forma gradual a redução e/ou perda do liquido sinovial e ainda, osteófitos centrais ou marginais, levando a um quadro de processo inflamatório crônico com esclerose articular ${ }^{7,8}$.

Observou-se também, que a degeneração articular progride de forma lenta, levando ao quadro 
sintomático de dor, rigidez e instabilidade articular, deformidades, edema e fraqueza muscular, com possibilidade de prejuízo à capacidade funcional e consequentemente, impacto na qualidade de vida dos pacientes $^{9,10}$.

Fatores como idade elevada, traumas repetitivos na articulação, excesso de peso, sobrecarga articular por determinadas práticas esportivas ou laborais, fraqueza muscular e hereditariedade são alguns aspectos relacionados ao desenvolvimento ou agravo dessa morbidade ${ }^{11}$.

Atualmente, o diagnóstico clínico da $\mathrm{AO}$ do joelho é realizado tomando como base quatro pontos principais: o quadro clínico apresentado pelo paciente, exame físico, os fatores predisponentes e a análise da condição radiológica dessa articulação que pode apresentar estreitamento de espaço intraarticular, osteofitose, esclerose do osso subcondral e formações císticas ${ }^{11-13}$.

De forma insidiosa e gradual ocorre o agravo do quadro clínico e funcional, tornando as habilidades funcionais e a qualidade de vida desses pacientes afetada direta ou indiretamente, seja em suas atividades cotidianas de lazer, laborais ou mesmo nas relações sociais ${ }^{14,15}$.

É preciso esclarecer ainda, que a capacidade funcional é conceituada como o potencial para o indivíduo cumprir as atividades cotidianas sem precisar de auxílio ${ }^{16}$, aspecto indispensável para a qualidade de vida ${ }^{17}$, que é definida como a percepção dos indivíduos sobre sua maneira de viver em um contexto mais amplo, dos princípios de cultura e valor, nos quais é observado os seus modelos e objetivos de vida, esperanças e ansiedades ${ }^{18}$.

Nesse sentido, este presente estudo justificase pela grande incidência desta doença, sobretudo pelo seu impacto social e pela ausência de estudos com essa temática nesse município, e assim, o objetivo desse estudo foi investigar o impacto da osteoartrose do joelho na capacidade funcional, bem como, na qualidade de vida dos pacientes atendidos no setor de Fisioterapia na cidade de Serra Talhada, Município de Pernambuco.

\section{MATERIAL E MÉTODO}

Trata-se de um estudo transversal do tipo descritivo e natureza quantitativa ${ }^{19}$. A amostra foi constituída por 26 pacientes atendidos em unidades municipais de fisioterapia no município pernambucano de Serra Talhada, no período de agosto a outubro de 2016. Os indivíduos foram recrutados por amostragem não probabilística por conveniência de acordo com critérios de elegibilidade relacionados ao estudo.

Foram incluídos no estudo pacientes com diagnóstico de osteoartrose de joelho (uni ou bilateral), de ambos os sexos, idade superior a 18 anos e classificação radiológica de Kellgren e
Lawrence entre grau I e IV. Pacientes portadores de outras doenças osteoarticulares (artrite reumatoide), pacientes que realizaram cirurgias no joelho, portadores de chikungunya e indivíduos com doenças degenerativas que alterassem as habilidades funcionais e aspectos da qualidade de vida (cardiopatias, neoplasias, doença de Parkinson) foram excluídos do estudo.

Os dados sociodemográficos foram coletados através de aplicação de questionários e escalas em local que garantisse a privacidade e sigilo das respostas dos participantes.

Para a avaliação da dor foi utilizado a Escala Analógica Visual (EVA) que gradua a intensidade do quadro álgico em leve, moderada e intensa através de uma escala em centímetros $(\mathrm{cm})$ que varia de zero centímetro (sem dor), seguindo de forma crescente até dez centímetros (dor insuportável) ${ }^{20}$.

A análise radiológica foi realizada por meio da classificação de Kellgren e Lawrence ${ }^{32}$ empregada apenas em projeção anteroposterior. É graduada em cinco estágios da osteoartrose, sendo o grau 0 : ausência de osteoartrose, grau I: estreitamento duvidoso do espaço articular, possível desenvolvimento de osteófitos, grau II: osteófitos definitivos, estreitamento questionável do espaço articular, grau III: osteófitos moderados, estreitamento definitivo, alguma esclerose, possível deformidade articular e o grau IV: osteófitos de grandes dimensões, estreitamento marcado, esclerose grave, deformidade articular estabelecida.

A capacidade funcional foi mensurada através do questionário específico WOMAC (Western Ontario McMaster Universities Osteoarthritis Index) que avalia as seguintes variáveis: dor, rigidez articular e habilidade funcional, classificando a funcionalidade do indivíduo para prática de atividades da vida diária (AVD), como: tomar banho, higiene pessoal, vestirse, uso dos sanitários, deambular, transferências, descer ou subir escadas. Com escore que varia de 0 96. Assim quanto maior a pontuação final da escala, pior a capacidade funcional ${ }^{21}$.

A estimativa dos aspectos relacionados a qualidade de vida se deu pela aplicação do questionário de Qualidade de Vida SF-36 (ShortForm 36) instrumento genérico composto por 36 itens, subdividido nas seguintes variáveis: capacidade funcional, dor, vitalidade, aspecto físico, saúde geral, aspectos sociais e emocionais, além da saúde mental. $\mathrm{O}$ escore é mensurado de 0 (pior qualidade de vida) a 100 (melhor qualidade de vida) ${ }^{22,23}$.

Os dados foram tabulados em planilhas do software Microsoft Excel 2013 e submetidos à análise estatística do tipo descritiva onde os dados foram expressos em tabelas e gráficos. O nível de significância estatística da relação entre as variáveis se deu quando $\mathrm{p} \leq 0,05$. 
O estudo foi submetido ao Comitê de Ética em Pesquisa da Fundação Francisco Mascarenhas/Faculdades Integradas de Patos, Paraíba, e aprovado com parecer $\mathrm{n}^{\circ} 1825580$, sendo assim o mesmo se encontra em consonância com a resolução 466/12 do CONEP/CEP.

\section{RESULTADOS}

A amostra foi caracterizada por indivíduos com média de idade de 63,4 anos, sendo $80,8 \%$ $(\mathrm{n}=21)$ do gênero feminino e $19,2 \%(\mathrm{n}=5)$ masculino. No estado civil, $50 \%(n=13)$ eram casados e $42,3 \%$ $(\mathrm{n}=11)$ eram viúvos/separados, em relação a cor, observou-se que a cor negra e parda representaram $77 \%(n=20)$ e a branca $23 \%(n=6)$. Os aposentados foram maioria com $65,3 \%(\mathrm{n}=17)$ e apenas $34,7 \%$ $(\mathrm{n}=9)$ ainda trabalhavam.

Ainda nesta amostra 84,4\% $(\mathrm{n}=22)$ eram sedentários e apenas $15,4 \%(n=4)$ ativos, com duas principais comorbidades, hipertensão arterial presente em $61,5 \%(n=16)$ dos indivíduos, seguida da diabetes mellitus que representou 19,2\% ( $\mathrm{n}=5)$ (Tabela 1).

Tabela 1. Dados sociodemográficos de pacientes com AO do joelho

\begin{tabular}{|c|c|c|}
\hline VARIÁVEL & $\mathbf{n}^{\circ}$ & $\%$ \\
\hline \multicolumn{3}{|l|}{ Gênero } \\
\hline Feminino & 21 & 80,8 \\
\hline Masculino & 05 & 19,2 \\
\hline \multicolumn{3}{|l|}{ Cor/Etnia } \\
\hline Negra & 14 & 54 \\
\hline Parda & 06 & 23 \\
\hline Branca & 06 & 23 \\
\hline \multicolumn{3}{|l|}{ Estado Civil } \\
\hline Casado & 13 & 50 \\
\hline Viúvo/Separado & 11 & 42,3 \\
\hline Solteiro & 02 & 7,7 \\
\hline \multicolumn{3}{|l|}{ Ocupação } \\
\hline Aposentado & 17 & 65,3 \\
\hline Ativo & 09 & 34,7 \\
\hline \multicolumn{3}{|l|}{ Atividade Física } \\
\hline Sedentário & 22 & 84,6 \\
\hline Ativo & 04 & 15,4 \\
\hline \multicolumn{3}{|l|}{ Comorbidades } \\
\hline Hipertensão A. & 16 & 61,5 \\
\hline Diabetes M. & 05 & 19,2 \\
\hline \multicolumn{3}{|l|}{ Idade } \\
\hline (Média; Min-Máx) & 63,4 & $(40-85)$ \\
\hline
\end{tabular}

Do mesmo modo, outros aspectos clínicos foram analisados, e assim, foi possível indicar que a 92\% ( $\mathrm{n}=24)$ dos indivíduos se apresentavam acima do peso ideal, com alteração postural no joelho, isto é $46,2 \%(n=12)$ com geno varo e apenas $11,5 \%(n=3)$ apresentavam geno valgo. $\mathrm{O}$ quadro sintomatológico estava presente em $61,5 \%(n=16)$ de forma bilateral e no exame radiológico, o grau II foi mais frequente, representando 42,2\% $(n=11)$ da amostra (Tabela 2).

Em relação a intensidade do quadro álgico do joelho, esta foi mensurada através da Escala Visual Analógica (EVA) onde pontuações de 0 a 2 indicam uma dor leve, enquanto que uma dor de intensidade moderada corresponde a pontuação de 3 a 7 e um quadro álgico de caráter intenso é graduado de 8 a 10 nesta escala. Todos os indivíduos relataram dor no joelho, entretanto observou-se que a maioria dos indivíduos, isto é, 14 (53,9\%) apresentaram um quadro doloroso intenso no joelho (Figura 1). A média na EVA foi de 7 onde 2 foi a graduação mínima e 10 a máxima.

\begin{tabular}{|c|c|c|}
\hline VARIÁVEL & $\mathbf{n}^{\circ}$ & $\%$ \\
\hline \multicolumn{3}{|l|}{ Condição Corporal (Kg/h²) } \\
\hline Sobrepeso & 11 & 42,5 \\
\hline Obesidade tipo I & 08 & 30,7 \\
\hline Obesidade tipo II & 04 & 15,4 \\
\hline Eutrófico & 02 & 7,6 \\
\hline Obesidade Mórbida & 01 & 3,8 \\
\hline \multicolumn{3}{|l|}{ Geno } \\
\hline Varo & 12 & 46,2 \\
\hline Normal & 11 & 42,3 \\
\hline Valgo & 03 & 11,5 \\
\hline \multicolumn{3}{|l|}{ Acometimento } \\
\hline Bilateral & 16 & 61,5 \\
\hline Unilateral & 10 & 38,5 \\
\hline \multicolumn{3}{|l|}{ Grau Radiológico } \\
\hline I & 07 & 27 \\
\hline II & 11 & 42,2 \\
\hline III & 07 & 27 \\
\hline IV & 01 & 3,8 \\
\hline
\end{tabular}

\section{Escala Visual Analógica}

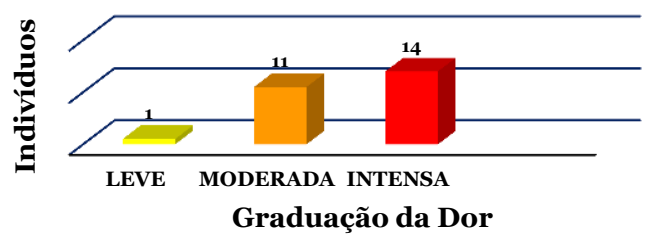

Figura 1: Intensidade do quadro álgico de pacientes com AO do joelho.

Em relação a capacidade funcional foi visto que diferentes atividades cotidianas apresentaram pontuações de distintos graus de dificuldade para os portadores de $\mathrm{AO}$ de joelho. Através do questionário WOMAC (Western Ontario McMaster Universities Osteoarthritis Index) específico para AO de joelho e quadril, foi possível mensurar quais AVDs que apresentaram maior dificuldade e limitação para os pacientes. Destaca-se que quanto maior a pontuação no questionário, maior é a dificuldade de execução daquela atividade.

Nesse sentido, observou-se que as AVDs caminhar em superfície plana e fazer compras com as respectivas pontuações de 31 e 38 tiveram menor dificuldade de execução para os portadores de AO de joelho. Enquanto que descer escada (65), calçar o sapato (69) e subir escada (69) foram as atividades com maior grau de dificuldade. A média geral foi $54,2 \pm 13,9$ indicando que a limitação se deu de forma moderada. Outras atividades como tomar banho, sentar no vaso sanitário e entrar/sair do carro, obtiveram pontuação de 54 , indicando um grau moderado de limitação (Figura 2).

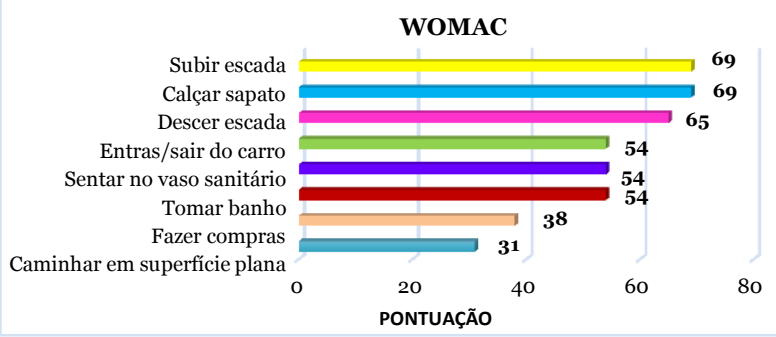

Figura 2: Pontuação do questionário WOMAC para atividades cotidianas 
Foi analisado ainda aspectos relacionados a qualidade de vida dos portadores de AO de joelho através do questionário SF-36 (Short- Form 36) onde a graduação varia de 0 a 100 e por consequência, pontuações mais próximas de 100 indicam melhor qualidade de vida. Dessa forma, aspectos como a capacidade funcional (10) e o estado geral de saúde (25) mostraram-se mais comprometidos nesses indivíduos, enquanto que saúde mental (58) e aspectos sociais (68) revelaram-se aspectos menos afetados pela OA de joelho (Figura 3). A pontuação média foi 41,2 sinalizando uma qualidade de vida moderada nesses indivíduos.

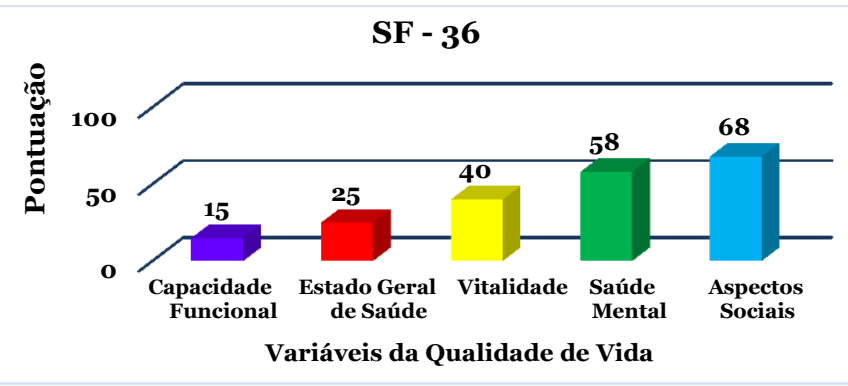

Figura 3: Pontuação dos aspectos relacionados a qualidade de vida.

As variáveis do questionário $\mathrm{SF}$ - 36 foram analisadas pela correlação de Pearson e mostraram correlação estatisticamente significativa $(p \leq 0,05)$, onde a vitalidade e os aspectos gerais de saúde obtiveram $p=0,014$, bem como, as variáveis de saúde mental e os aspectos sociais $p=0,018$. Pode-se inferir através destes resultados que estas variáveis da qualidade de vida são afetadas de forma interdependente pela $\mathrm{AO}$ de joelho.

DISCUSSÃO

O perfil sociodemográfico mostrou resultados semelhantes a um estudo ${ }^{24}$ que abordou a mesma temática, no qual apresentou amostra semelhante $(n=30)$ e foi observado que $70 \%$ da sua amostra foi constituída de mulheres com média de idade de 71 anos, resultado também encontrado em outro estudo ${ }^{13}$ onde $74,2 \%$ da amostra $(n=93)$ era do gênero feminino com média de idade de 60 anos. Dessa maneira, compreende-se que independentemente do tamanho da amostra, o gênero feminino na faixa etária acima dos 60 anos, lidera de forma indiscutível em números de acometimento.

Foi observado ainda que indivíduos acima de 60 anos comumente apresentam comorbidades, dessa forma hipertensão arterial $(61,5 \%)$ e diabetes mellitus $(19,2 \%)$ foram as principais encontradas. Nesse sentido, outro estudo com amostra bem maior $(n=355)$ também apontou que a hipertensão arterial foi a comorbidade mais prevalente com $44,1 \%$, seguida da diabetes mellitus com $12,8 \%$.

$O$ índice de pratica de atividade foi outro fator proeminente neste estudo, onde $84,4 \%(n=22)$ dos indivíduos eram sedentários, percentual semelhante em um estudo ${ }^{24}$ onde os inativos eram grande maioria $(73,3 \%)$ na amostra de 30 indivíduos. Dessa maneira, a literatura mostra que indivíduos com $\mathrm{OA}$ são desfavoráveis à pratica de atividade física pois relatam desconforto, medo de agravamento ou porque receberam instruções médicas previas para evitar o exercício. De qualquer forma, a condição funcional mais prejudicada foi observada nos sedentários, portanto o sujeito menos ativo tende a piora das condições psicofísicas e ao isolamento social, prejudicando assim sua qualidade de vida ${ }^{25,26}$.

$\mathrm{O}$ índice de massa corpórea (IMC) constatado neste estudo mostrou-se um fator preocupante, pois $92 \% \quad(\mathrm{n}=24)$ dos indivíduos estavam acima do peso ideal, entretanto o tamanho da amostra pode ter influenciado esse alto percentual, pois em estudo ${ }^{6}$ semelhante, uma amostra maior $(\mathrm{n}=355)$ revelou que a maioria, isto é, 140 indivíduos $(39,4 \%)$ apresentou-se eutrófica.

É importante ressaltar que pacientes com sobrepeso ou obesos apresentam tendência ao agravamento da condição clínica da OA de joelho, como a dor e a limitação funcional, consequentemente esses pacientes poderão apresentar prejuízos em sua qualidade de vida ${ }^{2,27}$. Confirmando a tese anterior, um artigo $^{28}$ encontrou correlação positiva, entre os resultados obtidos pelo WOMAC e o IMC, sinalizando relação entre o sobrepeso e evolução no agravo dos sintomas da OA de joelho.

Outro fato importante é que o joelho se destaca por ser uma estrutura comumente afetada por alterações degenerativas, como a osteoartrose que influência no alinhamento dessa articulação ${ }^{29}$. O maior surgimento do geno varo em 12 indivíduos $(46,2 \%)$ está em concordância com a literatura que observa essa deformidade com maior incidência na evolução da OA de joelho ${ }^{30,31}$. Entretanto, faz-se necessário o desenvolvimento de outros estudos uniformizando o método e instrumentos utilizados para essa análise.

Foi examinado também a condição radiológica por meio das incidências anteroposterior e perfil, observou-se que $42,2 \% \quad(n=11)$ apresentou grau II na classificação de Kellgren e Lawrence ${ }^{32}$, o que indica uma OA de joelho leve (possível estreitamento do espaço articular e osteófitos definidos). Há poucos relatos na literatura sobre a classificação radiológica da OA, entretanto, um estudo $^{29}$ também observou na sua amostra $(n=30)$ que a média dos indivíduos apresentou grau II nessa classificação.

Em outro estudo com 46 indivíduos foi constatado que $45 \% \quad(n=21)$ apresentou grau III predominante, revelando uma OA moderada, isto é, osteófitos moderados, estreitamento definitivo, alguma esclerose, possível deformidade $\operatorname{articular}^{33}$. 
Um $^{\text {artigo }^{34}}$ afirmou que não é regra haver uma correlação de proporção entre o grau de severidade dos sinais radiológicos e o quadro clínico, principalmente o doloroso.

Ressalta-se que a dor está associada ao aumento da pressão intraóssea pela congestão vascular do osso subcondral, sinovite, crescimento dos osteófitos, fibrose capsular, fraqueza e contratura muscular $^{35}$. Em um trabalho ${ }^{36}$ foi confirmado que a OA de joelho colabora para um quadro álgico importante e limitante nos sujeitos.

Dessa forma, foi relatado que a média de graduação na EVA foi 8, indicando que esses indivíduos compartilhavam de um quadro álgico intenso nos joelhos ${ }^{24}$. Neste estudo que teve objetivo semelhante ao anteriormente citado, observou-se que todos os indivíduos apresentavam dor significativa nos joelhos, mas a graduação média foi de 6,9 na EVA, revelando alto índice de dor nessa população.

Foi mensurado através do WOMAC a capacidade funcional de portadores de OA de joelho $(n=355)^{6}$, obtendo resultados semelhantes a este estudo, onde a AVD com menor limitação foi fazer compras (45), enquanto que a atividade descer escada (90) foi a que obteve resultado mais alto nesse questionário, indicando que esta é uma AVD onde os pacientes apresentam maior dificuldade. Um estudo ${ }^{28}$ avaliou 40 indivíduos através do WOMAC, e dessa forma também confirmam que subir $(67,5)$ e descer escada (60) são as atividades com mais queixas de dificuldade na opinião dos portadores de OA de joelho.

Um estudo Japonês revela que o portador de OA de joelho, de modo geral, apresenta redução de força (50 a 60\%) no músculo quadríceps femoral com possível contratura leve na extensão do joelho por atrofia e inibição artrogênica $^{37}$, além da diminuição do equilíbrio e propriocepção dessa articulação, em comparação a indivíduos sem essa morbidade. Isso explicaria porque as maiores limitações no WOMAC ocorreram em atividades que requerem a ativação principalmente do quadríceps femoral, como subir e/ou descer escada ${ }^{38,39}$.

Estudos apontam que pacientes com OA de joelho submetidos a alongamento para flexibilidade $\mathrm{e}$ a treino de fortalecimento da musculatura do quadríceps femoral, proporcionaram melhora expressiva na sua qualidade de vida ${ }^{40,41,42}$.

A literatura sinaliza ainda que a OA de joelho é avaliada como fator limitante da capacidade funcional, influenciando de forma direta na qualidade de vida de indivíduos acima de 60 anos, e observa que $98,6 \%$ apresentaram dor, com dificuldades relevantes na execução das atividades do cotidiano $^{6,43}$.

Dessa maneira, foi avaliada em um estudo ${ }^{13}$ a qualidade de vida de indivíduos $(n=93)$ e com objetivo semelhante a este estudo, observou que os aspectos capacidade funcional (60) e vitalidade (60) foram os mais afetados pela OA de joelho, porém diferentemente deste estudo as pontuações foram bem maiores. Ainda de acordo com o mesmo autor supracitado, os aspectos da qualidade de vida menos atingidos foram aspectos sociais $(68,8)$ e saúde mental (78).

\section{CONCLUSÃO}

Através deste estudo constatou-se que a OA de joelho gera, especialmente em mulheres acima dos 60 anos, quadro doloroso intenso, influenciando de forma negativa na capacidade funcional, tornando-se assim, fator limitante em determinadas AVDs dos indivíduos portadores desse processo patológico. Observou-se ainda que a capacidade funcional representa a independência cotidiana desses indivíduos, algo relevante para a saúde física e psicossocial, por essa razão essas limitações funcionais desencadeiam um grande impacto negativo sobre alguns aspectos que constituem a qualidade de vida.

Houve variáveis estatisticamente significativas $(\mathrm{p} \leq 0,05)$ na análise da qualidade de vida, onde a vitalidade e os aspectos gerais de saúde obtiveram $\mathrm{p}=0,014$, bem como, as variáveis de saúde mental e os aspectos sociais $\mathrm{p}=0,018$. Dessa maneira, pode-se concluir que estes aspectos da qualidade de vida são influenciados de forma conjunta pela AO de joelho. Contudo, não houve correlações estatisticamente significativas entre as demais variáveis estudadas.

Este estudo abre precedente para que outras pesquisas sejam realizadas com amostras maiores, comparando populações ativas e sedentárias ou ainda, analisando isoladamente determinadas faixas etárias acima de 60 anos de idade, e dessa forma, permitir correlacionar outras variáveis da OA de joelho. Com um número maior e variado de estudos sobre essa temática será possível entender com mais detalhes as implicações dessa doença na qualidade de vida, possibilitando uma atuação mais eficaz da fisioterapia baseada essencialmente em evidências e respeitando as características de cada população.

\section{REFERÊNCIAS}

1. Xavier APS. Acupuntura em pacientes com osteoartrite de joelho. [monografia]. Montes Claros: Faculdade de Educação, Ciência e Tecnologia; 2007

2. Vasconcelos KSS, Dias JMD. Relação entre intensidade de dor e capacidade funcional em indivíduos obesos com osteoartrite de joelho. Rev bras fisioter. 2006;10(2):213-18.

3. Silveira MM, Sachetti A, Vidmar MF, Venâncio G, Tombini DK, Sordi $\mathrm{S}$ et al. Perfil epidemiológico de idosos com osteoartrose. R Ci méd biol. 2011;9(3):212-15. 
4. Menken M, Munsat TL, Toole JF. The global burden of disease study: implications for neurology. Arch neurol. 2000;57(3):418-20.

5. Figueiredo Neto EM, Queluz TT, Freire BFA. Atividade física e sua associação com qualidade de vida em pacientes com osteoartrite. Rev Bras Reumatol. 2011;51(6):544-49.

6. Cassettari MR. Osteoartrose em joelhos como fator limitante para a qualidade de vida em idosos [dissertação]. São Paulo: Programa de PósGraduação em Saúde Coletiva - UNIFESP; 2008.

7. Silva F, Goes P. Efeitos da Fisioterapia Aquática na dor e função musculoesquelética de idosos com osteoartrite de joelho [monografia]. Belo Horizonte. Curso de Fisioterapia - Universidade Federal de Minas Gerais UFMG; 2008.

8. Gomes WF. Impacto de um programa estruturado de fisioterapia aquática em idosas com osteoartrite de joelho [dissertação]. Belo Horizonte: Programa de Pós-Graduação em Ciências da Reabilitação, Universidade Federal de Minas Gerais - UFMG; 2007.

9. Page CJ, Hinman RS, Bennell KL. Physiotherapy management of knee osteoarthritis. Int $\mathrm{J}$ Rheum Dis. 2011;14(2):145-51.

10. Silva MP, Barros CAM. Benefícios de um programa de exercícios funcionais no tratamento da osteoartrite de joelho. Saúde. 2012; 1(1):23-42.

11.Zacaron KAM, Dias JMD, Abreu NS, Dias RC. Nível de atividade física, dor e edema e suas relações com a disfunção muscular do joelho de idosos com osteoartrite. Rev bras fisioter. 2006;10(3):279-84.

12. Matos DR, Araujo TCCF. Qualidade de vida e envelhecimento: questões específicas sobre osteoartrose. Psico Estud 2009;14(3):511-18.

13. Araujo ILA. Qualidade de Vida e Independência Funcional em Portadores de Osteoartrite do Joelho. Bahia [dissertação]. Salvador: Escola Bahiana de Medicina e Saúde Pública da Bahia Bahiana; 2014.

14. Marchon RM, Cordeiro RC, Nakano MM. Capacidade Funcional: estudo prospectivo em idosos residentes em uma instituição de longa permanência. Rev bras geriatr gerontol. 2010;13(2):203-14.

15. Sutbeyaz ST1, Sezer N, Koseoglu BF, Ibrahimoglu F, Tekin D. Influence of knee osteoarthritis on exercise capacity and quality of life in obese adults. Obesity (Silves Spring). 2007; 15(8):2071-76.

16. Farinati PTV. Avaliação da autonomia do idoso: definição de critérios para uma abordagem positiva a partir de um modelo de interação saúdeautonomia. Arq Geriatr Gerontol. 1997;1:1-9.

17. Alves LC, Leimann BCQ, Vasconcelos MEL, Carvalho MS, Vasconcelos AGG, Fonseca TCO et al. Influência das doenças crônicas na capacidade funcional de idosos. Cad Saúde Pública 2007;23(8):1924-30.

18. OMS. Organização Mundial da Saúde. Divisão de saúde mental, grupo whoqol. 1997. Disponível em: http://www.ufrgs.br/psiquiatria/psiq/whoqol 1.html. Acesso em 20 ago, 2016.

19. Appolinário F. Metodologia da ciência: filosofia e prática da pesquisa. 2. ed. São Paulo: Cengage; 2012.

20. Scott J, Huskisson EC. Vertical or horizontal visual analogue scales. Ann Rheum Dis. 1979;38(6):560.

21. Fernandes MI. Tradução e validação do questionário de qualidade de vida específico para osteoartrose WOMAC (Western Ontario McMaster Universities) para a língua portuguesa [dissertação]. São Paulo: Faculdade de MedicinaUNIFESP; 2003.

22. Yilmaz F, Sahin F, Ergoz E, Deniz E, Ercalik C, Yucel SD et al. Quality of life assessments with SF 36 in different musculoskeletal diseases. Clin rheumatol. 2008;27(3):327-32.

23. Turner-Bowker DM, DeRosa MA, Ware JE Jr. SF-36® Health Survey. In S. Boslaugh (Ed.), Encycl Epidemiol, v. 2. Thousand Oaks, CA: Sage Publications; 2008.

24. Lopes D, Santos SL. Avaliação da funcionalidade em idosos com osteoartrose de joelho [monografia] Bragança Paulista: Curso de Fisioterapia, Universidade São Francisco - USF; 2007.

25. Hootman JM, Macera CA, Ham SA, Helmick CG, Sniezek JE. Physical activity levels among the general US adult population and in adults with and without arthritis. Arthritis Rheum. 2003;49(1):129-35.

26. Steultjens MP, Dekker J, Bijlsma JW. Avoidance of activity and disability in patients with osteoarthritis of the knee: the mediating role of muscle strength. Arthritis Rheum. 2002; 46(7):1784-88.

27. Messier SP, Gutekunst DJ, Davis C, DeVita P. Weight loss reduces knee-joint loads in overweight and obese older adults with knee osteoarthritis. Arthritis Rheum. 2005;52(7):2026-32.

28. Alves JC, Bassitt DP. Qualidade de vida e capacidade funcional de idosas com osteoartrite de joelho. Einstein 2013;11(2):209-15.

29. Sencovici L. Análise postural e atividade eletromiográfica em pacientes com osteoartrite de joelho [dissertação]. Faculdade de Medicina, Universidade de São Paulo (USP); 2009.

30. Sharma L, Kapoor D, Issa S. Epidemiology of osteoarthritis: an update. Cur Opin Rheumatol. 2006; 18(2):147-56.

31. Camanho GL. Tratamento da osteoartrose do joelho. Rev Bras Ortop. 2001; 36(5):135-40.

32. Kellgren JH, Lawrence JS. Radiological 
assessment of osteoarthritis. Ann Rheum Dis 1957; 16:494-501.

33. Alfredo PP. Eficácia da laserterapia de baixa intensidade associada a exercícios em pacientes com osteoartrose de joelho: estudo randomizado e duplo-cego [tese]. São Paulo: Faculdade de Medicina, Universidade de São Paulo - USP; 2011.

34. Thumboo J, Chew LH, Lewin-Koh SC. Socioeconomic and psychosocial factors influence pain or physical function in Asian patients with knee or hip osteoarthritis. Ann Rheum Dis. 2002;61(11):1017-20.

35. Camanho GL, Imamura M, Arendt-nielsen L. Gênese da dor na artrose. Rev Bras Ortop, 2011;1(46):7-14.

36. Chapple C. Physiotherapy for osteoarthritis of the knee: predictors of outcome at one year [tese]: Nova Zelândia: Universidade de Otago; 2011.

37. Hassan B, Mockett S, Doherty M. Static postural sway, proprioception, and maximal voluntary quadriceps contraction in patients with knee osteoarthritis and normal control subjects. Ann Rheum Dis. 2001;60(6):612-18.

38. Watanabe $\mathrm{H}$, Urabe K, Takahira N, Ikeda N, Fujita M, Obara S et al. Quality of life, knee function, and physical activity in Japanese elderly women with early-stage knee osteoarthritis. J Orthop Surg (Hong Kong). 2010;18(1):31-4.

39. Silva ALP, Imoto DM, Croci AT. Estudo comparativo entre a aplicação de crioterapia, cinesioterapia e ondas curtas no tratamento da osteoartrite de joelho. Acta ortop bras. 2007; 15(4):204-9.

40. Ağlamiş B, Toraman NF, Yaman H. Change of quality of life due to exercise training in knee osteoarthritis: SF-36 and WOMAC. J Back Musculoskeletal Rehabil. 2009;22(1):43-5, 47-8.

41. Imoto AM, Peccin MS, Trevisani VFM. Exercícios de fortalecimento de quadríceps são efetivos na melhora da dor, função e qualidade de vida de pacientes com osteoartrite do joelho. Acta Ortop Bras. 2012;20(3):174-79.

42.Pedrinelli A, Garcez-Leme LE, Nobre RSA. O efeito da atividade física no aparelho locomotor do idoso. Rev bras ortoped. 2009;44(2):96-101.

43. Majani G, Giardini A, Scotti A. Subjective impact of osteoarthritis flare-ups on patients' quality of life. Health Qual Life Outcomes. 2005;3:14.
CONFLITO DE INTERESSES

Os autores declaram não haver conflitos de interesse.

\section{AUTOR PARA CORRESPONDENCIA}

\section{Ruan Emerson Rodrigues}

ruanfisioterapeuta@hotmail.com

Submetido em 04/02/2019

Aceito em 24/05/2019 